\title{
CONTINUOUS FUNCTIONS OF HERMITIAN OPERATORS ${ }^{1}$
}

\author{
P. R. HALMOS
}

ABSTRACT. Theorem: every normal operator is a continuous function of a Hermitian one. Corollary: every normal operator on a separable Hilbert space is the sum of a diagonal operator and a compact one.

THEOREM. Every normal operator is a continuous function of a Hermitian one.

COROLlaRY. Every normal operator on a separable Hilbert space is the sum of a diagonal operator and a compact one.

Any two commutative Hermitian operators are Borel functions of some Hermitian operator; the version of the theorem in which "continuous" is replaced by "Borel" is old. Both the Borel and the continuous versions extend to countable sets of commutative Hermitian operators, with no additional effort. The present version is an easy consequence of well known Hilbert space techniques, together with an elegant fact of general topology. The result, with a similar but different proof, is implicit in a construction of Schwartz [6, pp. 15-16]. Much is known about normal operators, but not everything. The theorem is presented here in the hope that it may yield some new information. The basis of the hope is the derivation of the corollary from the theorem.

The version of the corollary in which "normal" is replaced by "Hermitian" is the Weyl-von Neumann theorem; till quite recently the extension to the normal case was an open problem [4]. The first solution is due to I. D. Berg [1]. The proof below is shorter, and it may perhaps be considered more translucent.

PROOF OF THE THEOREM. Every compact metric space is a continuous image of the Cantor set [5, $\S 41$, VI]. If, in particular, $\Lambda$ is a non-empty compact subset of the complex plane and $\Gamma$ is the Cantor set in the unit interval, then there exists a continuous function $\dot{\varphi}$ from $\Gamma$ onto $\Lambda$. The elegant fact from general topology enters here: it is the existence of a Borel cross section. That is: there exists a Borel function $\psi$ from $\Lambda$ into $\Gamma$ such that the composition $\varphi \circ \psi$ is the identity on $\Lambda$ [5, $\S 43, \mathrm{IX}]$. (Short

Received by the editors February 3, 1971.

AMS 1970 subject classifications. Primary 47B15.

${ }^{1}$ Research supported in part by a grant from the National Science Foundation. 
proof for the case at hand: for each $z$ in $\Lambda$, let $\psi(z)$ be the infimum of the numbers $x$ in $\Gamma$ for which $\varphi(x)=z$. If $z_{n} \rightarrow z$, then the continuity of $\varphi$ implies that the limit of each convergent subsequence of $\left\{\psi\left(z_{n}\right)\right\}$ is greater than or equal to $\psi(z)$. It follows that $\psi(z) \leqq \lim \inf _{n} \psi\left(z_{n}\right)$, so that $\psi$ is lower semicontinuous, and therefore of Baire class 1.)

Suppose that $\mu$ is a positive finite Borel measure with support in $\Lambda$, and write $\nu=\mu \circ \psi^{-1}$ for the induced measure in $\Gamma$. The mapping $T$ from $L^{2}(\nu)$ into $L^{2}(\mu)$ defined by $T g=g \circ \psi$ is an isometry. (This is standard; all that is needed to verify it is to look at the expressions for $\|g\|^{2}$ and $\|T g\|^{2}$ and to recall the way to change variables in Lebesgue integrals [2, §39].) The isometry $T$ maps $L^{2}(v)$ onto $L^{2}(\mu)$; indeed the inverse of $T$, which in this situation is the same as the adjoint of $T$, is defined by $T^{*} f=f \circ \varphi$. Since $\varphi \circ \psi$ is the identity on $\Lambda$, the equation $T T^{*} f=f$ is obvious. Since $\psi \circ \varphi$ is, in general, not the identity on $\Gamma$, the equation $T^{*} T g=g$ is true for a different reason; it follows from the isometric character of $T$.

The position operator on $L^{2}(\mu)(f(z) \mapsto z f(z))$ is the transform by $T^{*}$ of multiplication by $\varphi$ on $L^{2}(v)$. Proof: $\left(T\left(\varphi \cdot T^{*} f\right)\right)(z)=(T(\varphi \cdot(f \circ \varphi)))(z)$ $=((\varphi \cdot(f \circ \varphi)) \circ \psi)(z)=\varphi(\psi(z)) \cdot f(\varphi(\psi(z)))$.

The spectral theorem says that every normal operator is the direct sum of position operators [3]. More precisely: to within unitary equivalence every normal operator $A$ can be obtained as follows. Fix a non-empty compact set $\Lambda$ in the plane, let $\mu$ vary over an arbitrary set of positive finite Borel measures in $\Lambda$, and form the direct sum of the corresponding position operators. As $\mu$ varies, $v$ varies. The direct sum of the corresponding position operators $(g(x) \mapsto x g(x))$ on the $L^{2}(v)$ 's is a Hermitian operator $B$ (because $\Gamma$ is a subset of the real line). Since $A=\varphi(B)$, the proof of the theorem is complete.

PROOF OF THE COROLLARY. In view of the theorem, it is sufficient to prove that if $B$ is a Hermitian operator on a separable Hilbert space, and if $\varphi$ is a complex-valued continuous function on the spectrum of $B$, then $\varphi(B)$ is the sum of a diagonal operator and a compact one.

By the Weyl-von Neumann theorem, $B=D+C$, where $D$ is diagonal and $C$ is compact. Extend $\varphi$ to a continuous function (into the complex plane) on a compact set that includes the spectra of both $B$ and $D$. The Weierstrass approximation theorem implies that there exists a sequence $\left\{p_{n}\right\}$ of (complex) polynomials that converges uniformly to (the extended) $\varphi$; it follows that $p_{n}(B) \rightarrow \varphi(B)$ and $p_{n}(D) \rightarrow \varphi(D)$ in the norm. If $C_{n}=$ $p_{n}(B)-p_{n}(D)$, then each $C_{n}$ is compact, and the sequence $\left\{C_{n}\right\}$ converges in the norm to a (necessarily compact) operator $K$. Since $\varphi(D)$ is diagonal (any orthonormal basis that diagonalizes $D$ does the same for each $p_{n}(D)$ ), and since $\varphi(B)=\varphi(D)+K$, the proof of the corollary is complete. 
ReMARK. Is the theorem true for arbitrary $C^{*}$ algebras? (Question via J. P. Williams.) The answer is no. Indeed, if $\Lambda$ is a non-empty compact subset of the complex plane, then the identity mapping $(z \mapsto z)$ on $\Lambda$ is an (obviously normal) element of $C(\Lambda)$. The theorem for $C(\Lambda)$ would imply the existence of a Hermitian (i.e., real-valued) continuous function $\psi$ on $\Lambda$ and the existence of a continuous function $\varphi$ on $\psi(\Lambda)$ such that $\varphi(\psi(z))=z$ for all $z$ in $\Lambda$. Under these circumstances $\psi$ is one-to-one, and, consequently, $\Lambda$ is homeomorphic to a subset of the real line. If $\Lambda$ is chosen to make this impossible (e.g., if $\Lambda$ is the perimeter of a circle), then no such $\psi$ can exist.

\section{REFERENCES}

1. I. D. Berg, An extension of the Weyl-von Neumann theorem to normal operators, Trans. Amer. Math. Soc. 160 (1971), 365-371.

2. P. R. Halmos, Measure theory, van Nostrand, New York, 1950. MR 11, 504.

3. What does the spectral theorem say?, Amer. Math. Monthly 70 (1963), 241-247. MR 27 \#595.

4. —_ Ten problems in Hilbert space, Bull. Amer. Math. Soc. 76 (1970), 887-933.

5. K. Kuratowski, Topology. Vol. II, Academic Press, New York, 1968. MR 41 \#4467.

6. J. T. Schwartz, $W^{*}$-algebras, Gordon and Breach, New York, 1967. MR 38 \#547.

Department of Mathematics, Indiana University, Bloomington, Indiana 47401 